\title{
Mathilde Anquetil \\ DELF B2 et Portfolio, une expérience de coopération pour une certification en contexte
}

\begin{abstract}
This paper analyses an experiment in collaboration between the ELP and DELF (official certification in the French language) for students at B2 level. The speaking test is based on the presentation and discussion of the language biography and project work collected in the dossier. The lively interaction between examiner and test taker, as authentic social agents, fulfils the action-oriented approach of the CEFR. The ELP works as a mediation tool for an ethical cooperation between the local educational system and an international agency, promoting both plurilingualism and intercultural dialogue. Most of the literature on portfolios and assessment (review in Quingua 2010) focuses on writing development, while our experiment concerns speaking skills embedded in social interaction with a portfolio-based final test.
\end{abstract}

Mots-clés: reconnaissance ; certificatio ; interaction sociale ; contextualisation standardisation

Mathilde Anquetil: Dipartimento Studi sul Mutamento Sociale, Istituzioni Giuridiche e Comunicazione, Via Don Minzoni 2, 62100 Macerata, Italie. E-mail: mathilde.anquetil@unimc.it

\section{Introduction}

Le portfolio Européen des langues (PEL) a pour vocation la promotion d'une gestion autonome de son plurilinguisme par un apprenant/acteur social qui construit sa biographie langagière et interculturelle. Mais si la fonction pédagogique du PEL est un acquis, sa fonction de documentation et de présentation doit faire l'objet d'une attention majeure car une fois le PEL nourri de l'expérience des langues et des cultures, il reste à lui trouver un lecteur externe avisé. La présentation officielle du PEL annonce que "les occasions sont nombreuses de présenter un PEL aux institutions éducatives et aux employeurs”. Or le PEL manque d'une instance de réception dans la réalité sociale, une instance qui retraduirait pour le public 
les valeurs portées par l'approche didactique portfolio (Kohonen 2001) en évaluation certificative, puis en diplôme.

Par ailleurs les certifications représentent un outil puissant d'application du CECR (Conseil de l'Europe 2001) dans ses fonctions de standardisation des niveaux de compétences linguistiques, elles renforcent la capitalisation et la mobilité des apprenants locuteurs, mais au sein de l'université des doutes surgissent quant à la pertinence et à la compatibilité des certifications externes au vu des finalités universitaires (Anquetil et Jamet 2010).

La recherche-action que l'on présentera a impliqué le Centro Linguistico d'Ateneo (CLA) de l'Université de Macerata, l'Alliance Française locale (San Benedetto del Tronto) et le Centre Pilote des certifications de l'Ambassade de France en Italie, pour la mise en place expérimentale d'un dispositif de certification de français DELF niveau B2 pour un public universitaire, joignant aux épreuves centralisées par le CIEP, la présentation du PEL, modèle CERCLES, comme document de base pour susciter l'interaction orale entre l'examinateur et le candidat.

Pour l'épreuve orale individuelle (documents en annexe), le candidat est invité à présenter sa biographie langagière puis à exposer avec la partie dossier du PEL une recherche personnelle menée lors de son apprentissage: un dossier de presse, une critique personnelle de livre ou de film, un compte-rendu de séjour d'étude ou de stage dans un pays francophone avec Erasmus, une recherche liée aux études en cours ... L'interaction avec l'examinateur s'enchaîne naturellement à l'exposé par demande de précisions et échange d'opinions.

Cette expérimentation apporte des éléments valorisant la fonction du Cadre Européen et du PEL dans l'enseignement universitaire.

\section{Pour une réception dynamique de la politique linguistique européenne}

\subsection{Problèmes de réception à l'université des outils de politique linguistique européenne}

À l'instar du programme de mobilité Erasmus, dispositif fortement voulu par les institutions européennes qui a introduit de profondes mutations dans le monde universitaire, le CECR et son corollaire le PEL, tous deux outils de la politique linguistique européenne, viennent bouleverser l'enseignement des langues à l'université. L'académie a souvent réagi de façon assez réticente : elle a d'abord ignoré un dispositif étranger à son habitus en le considérant comme non pertinent par rapport à ses propres objectifs. Le document a ainsi été longtemps consi- 
déré comme relevant de l'enseignement pour d'autres publics (adultes puis scolaires), pour des objectifs instrumentaux en deçà des qualifications de l'enseignement universitaire. C'est souvent par le biais des certifications externes que le CECR s'est introduit dans l'enceinte universitaire, mais le plus souvent réduit au tableau d'échelonnage de niveaux de compétences communicatives, et dans les institutions para-académiques que sont les centres linguistiques. Les agences de certifications dites externes ou étrangères (par rapport à l'université) qui se sont emparées plus rapidement de l'outil car associées à son élaboration, sont alors apparues comme potentiellement concurrentielles par rapport au pouvoir d'évaluation-certification des académies. Cependant dans ces mêmes centres linguistiques universitaires, il s'est développé un courant visant à une appropriation du CECR en vue de la création de certifications internes et de la mise au point de déclinaisons du portfolio adaptées aux exigences universitaires, comme le PEL du CercleS. Ces deux options sont souvent envisagées comme antagonistes autour d'enjeux de pouvoir symbolique et marchand.

Or notre projet vise à une collaboration fructueuse des institutions en jeu en particulier par l'apport du patrimoine propre à l'université : ses finalités culturelles et humanistes, ses compétences scientifiques interdisciplinaires, la particularité de son enseignement fondé sur le lien avec la recherche et sans cesse soumis à la réflexion critique, ce qui lui interdit toute démarche purement applicationniste.

L'université ne peut se retrancher face à la forte demande sociale pour des certifications standardisées qui témoigne, au-delà de tendances sociétales de capitalisation individuelle du savoir, d'une crise de confiance par rapport à ses propres modalités d'enseignement et de certification. Les certifications externes sont puissantes par le crédit qui leur est octroyé sur le marché du travail, elles apportent indéniablement une plus-value sur un CV et sont donc porteuses d'un crédit social que les universités ne sont pas toujours en mesure de garantir. ${ }^{1}$

Toutefois l'académie ne saurait non plus déléguer sans contrepartie son pouvoir certifiant par simple démarche d'externalisation acritique, ne serait-ce qu'au vu des coûts supplémentaires à la charge de ses usagers. Une des voies d'issue de ce conflit larvé est de tenter de collaborer activement à la formulation des modalités de certification conjointes. Comme l'indique Danielle Lévy, ex-directrice du CLA de Macerata:

1 Voir cependant le crédit croissant accordé aux diplômes universitaires de langues comme le CLES en France, le système UNIcert en Allemagne, la Certification de l'État Grec en Langues Étrangères, les diplômes universitaires d'italien langue étrangère. etc. qui gagneraient encore en crédibilité par un système de label de qualité CercleS. 
L'université doit être non seulement capable de s'adapter aux changements économiques et sociaux, mais de les théoriser, de les anticiper. En cela elle devrait être à même aussi bien de se servir de ces modèles sous la pression du monde extérieur que de les dépasser, dans la mesure où ces modèles constituent non seulement des instruments mais des objets de connaissance à construire et à parfaire (Lévy 2001 : 45).

\subsection{Perspective institutionnelle sur les certifications}

On fait donc le pari que les agences de certifications ont à gagner en se confrontant à la perspective critique de l'université sur leurs produits, grâce à son ancrage territorial qui la place du côté des usagers et grâce à son expertise pluridisciplinaire.

Du point de vue de la sociologie des échanges linguistiques, Pierre Bourdieu (1982) a montré que toute parole est évaluée sur un "marché linguistique" et que les institutions éducatives tendent à convertir en normes universelles les compétences qu'elles imposent comme seules légitimes, s'autorisant ainsi à prélever au passage un "profit de distinction". Ces considérations s'appliquent tout particulièrement à la situation d'examen où a lieu un échange y compris pécuniaire pour légitimer (à son niveau) la parole du candidat, à condition qu'il se soumette à la situation d'énonciation et aux canons linguistico-culturels imposés. Ni l'université, ni les agences de certification ne peuvent se soustraire à cette analyse démystifiante de leur activité. Cependant la conscience critique ne doit pas pour autant mener à l'inhibition de l'action éducative, elle engage au contraire à prendre les mesures aptes à réduire la "violence symbolique" exercée par les institutions et à ancrer toute opération dans un cadre de valeurs démocratiques.

Du point de vue de l'analyse institutionnelle des processus éducatifs, les certifications internationales sont marquées par leur provenance. Le CIEP comme agence ministérielle œuvre essentiellement sur le segment final du processus d'apprentissage et tend à se concentrer sur le seul calibrage de la matière verbale produite par des apprenants dont il n'a à charge ni la motivation, ni le développement langagier. L'Alliance Française se conçoit comme relais local des politiques de "rayonnement de la France": ainsi les apprenants de toutes nationalités y étudient-ils sur les mêmes manuels français à diffusion internationale. Ils ne sont pas surpris de retrouver dans la certification cette même orientation francocentrée.

Mais l'université ne peut adopter la perspective du candidat individuel qui se soumet volontairement au dispositif étranger en vue d'une capitalisation à des fins personnelles. Si l'université accueille en son sein une instance étrangère, elle imprime forcément une autre signification à la transaction. D'une part elle réinterprète le dispositif selon ses propres intérêts, qui peuvent être de limiter le pou- 
voir du personnel préposé à l'enseignement communicatif, ou de limiter ses propres dépenses en renvoyant le coût de la certification à l'usager. Mais en tant qu'institution nationale solidaire par rapport à ses usagers, l'université est aussi porteuse d'une instance de médiation culturelle. Elle est en position et en mesure de porter un regard critique sur les produits proposés, de s'opposer aux ethnocentrismes de l'autre, d'aménager un espace tiers d'action et de recherche en commun.

La prise de conscience des enjeux de pouvoir lors des tests a engendré un courant d'études sous le nom de Critical Language Testing et l'adoption d'une charte éthique dans l'exercice de la profession (Shohamy 2001). La déontologie d'une université publique repose sur d'autres fondements à la fois scientifiques et républicains mais ces études la concernent, en particulier dans la réflexion autour du pouvoir rétroactif (washback) des tests sur les processus éducatifs. On considérera en particulier l'émergence du concept de rights of test takers, la notion de test taker comprenant dans un sens élargi tous les acteurs que le test affecte.

\footnotetext{
What are the rights of the test takers? The "test taker" is defined here in a broader perspective rather than the individual who is taking the test. It is perceived as all those who are affected by the orst results - for goog or for bad. Thus, it includes the person who is actually taking the test, the teacher whose students are being tested, the principal whose school is being tested, and the parents whose children are taking a test. (Shohamy 2001: 156)
}

La notion même de "validité" du test englobe ses conséquences sociales selon Tim McNamara (McNamara and Roever 2006, McNamara 2010) qui développe pour les langues les analyses du psychologue américain Samuel Messick (Messick 1989).

\subsection{Pour un renouvellement de la réception du CECR}

L'introduction du CECR à l'université a eu le mérite de donner une assise aux compétences communicatives trop souvent marginalisées dans les curricula, mais la définition fonctionnelle des référentiels de niveaux et l'apparition des opérateurs externes de certification ont parfois eu pour conséquence un certain désengagement de la recherche en didactique des langues pour le public universitaire. L'enseignement communicatif relégué aux Centres Linguistiques tend, dans l'esprit des gestionnaires, à passer au rang de "services". L'enseignant y perdrait de son autonomie pour devenir tuteur-animateur d'une didactique prédéfinie. 
Les Centres Linguistiques sont souvent promoteurs de projets TICE (Technologies de l'Information et de la Communication pour l'Education), visant à confectionner des tests et formations en ligne, qui ont certes une valeur d'instruments d'appoint, mais où l'étudiant n'est plus pris en considération comme acteur social complexe. En l'absence de dispositifs hybrides (blended) avec tutorat et alternance entre cours en présentiel et auto-apprentissage, le destinataire de ces produits est souvent réduit à la seule catégorie d'apprenant fiché sur un niveau de A2 à C2, auquel on prescrit un certain nombre d'exercices qui n'ont rien d'interactif au sens où l'entend le CECR. Cela ne correspond pas à la conception de l'apprenant comme intercultural speaker dont l'aspiration n'est pas de s'assimiler au locuteur natif (Byram et Zarate 1997). On observe de façon générale, une certaine tendance vers la décontextualisation de l'enseignement, le retour à des pédagogies béhavioristes, la disparition de la médiation dynamique du groupe classe, l'atomisation de l'apprenant, l'automatisation des procédures d'évaluation.

Il s'agit là de conséquences d'une lecture extrêmement réductive du CECR.

\section{Pour une pleine prise en compte de la perspective actionnelle promue par le CECR}

\subsection{De l'apprenant à l'acteur social}

Rappelons l'approche retenue par le CECR pour ce qui est de l'usage général et de l'apprentissage des langues :

La perspective privilégiée ici est [...] de type actionnel en ce qu'elle considère avant tout l'usager et l'apprenant d'une langue comme des acteurs sociaux ayant à accomplir des tâches (qui ne sont pas seulement langagières) dans des circonstances et un environnement donnés, à l'intérieur d'un domaine d'action particulier. Si les actes de parole se réalisent dans des activités langagières, celles-ci s'inscrivent elles-mêmes à l'intérieur d'actions en contexte social qui seules leur donnent leur pleine signification. Il y a «tâche » dans la mesure où l'action est le fait d'un (ou de plusieurs) sujet(s) qui y mobilise(nt) stratégiquement les compétences dont il(s) dispose(nt) en vue de parvenir à un résultat déterminé. La perspective actionnelle prend donc aussi en compte les ressources cognitives, affectives, volitives et l'ensemble des capacités que possède et met en œuvre l'acteur social. (Conseil de l'Europe 2001: 15)

Dans cette optique, la tâche de base accomplie par l'apprenant en situation d'évaluation, est de produire une prestation langagière lui permettant d'obtenir un diplôme. Cependant si l'on veut examiner une performance représentative de 
réelles compétences communicatives il importe que cette transaction s'inscrive dans un contexte tel que l'interaction discursive produise une signification sociale à un deuxième niveau, celui du dialogue entre deux personnes mettant en jeu leurs "ressources cognitives, affectives et volitives".

$\mathrm{Au}$ premier niveau il y a transaction entre deux acteurs sociaux qui s'en tiennent à une catégorisation fonctionnelle: un examinateur chargé de faire produire de la matière verbale à calibrer et un candidat en position basse qui se soumet aux contraintes communicatives imposées. Au deuxième niveau il y a une interaction entre un locuteur natif expert dont la vocation professionnelle est d'ouvrir sa communauté de langage aux non-natifs, et un locuteur qui voudrait participer à la communauté de langage des natifs, et francophones en général, au terme d'une période d'apprentissage.

Cette deuxième catégorisation du candidat est particulièrement représentative pour le niveau B2, supérieur au niveau seuil de la compétence fonctionnelle, qui suppose un véritable engagement pour s'approprier d'aspects linguisticoculturels plus fins de la langue, et qui permet aussi d'accéder à une nouvelle expression de soi dans l'autre langue ; c'est un niveau où les étudiants commencent à dire qu'ils réussissent à "penser dans l'autre langue”, où ils ont lu une vaste gamme de textes authentiques, eu des contacts avec des locuteurs, où ils se sont créé une expérience sociale dans l'autre langue. Ils sont en train de se créer une voix personnelle dans l'autre langue.

La voix personnelle est l'expression d'une persona, c'est-à-dire d'une identité et d'une perspective sur le monde qui lui permettent d'agir comme individu plurilingue et pluriculturel. L'apprentissage d'une langue, loin d'être simplement l'acquisition d'un code linguistique, devient la recherche de nouvelles possibilités expressives pour se faire un style personnel dans chaque langue (Kern et Liddicoat 2008).

Or c'est à ce deuxième niveau d'interaction sociale que les activités langagières suscitées dans les épreuves ne sont pas toujours à la hauteur des exigences qualitatives.

\subsection{Les excès de la standardisation}

Le point de vue psychométrique a tendance à dominer la réflexion sur l'évaluation de la production orale : on remarque que les agences de certification tendent à centrer leurs propres études sur la fiabilité des critères et processus d'évaluation pour améliorer leur dispositif. Tel était l'objectif du séminaire international pour le calibrage de productions orales en 5 langues sur les 6 niveaux de l'échelle du 
CECR au CIEP à Sèvres en juin 2008, réunissant les opérateurs européens du secteur. $^{2}$

Les situations de production orale utilisées pour les opérations de calibrage étaient standardisées pour toutes les langues, dans le souci d'harmoniser l'interprétation des productions, mais les situations de communication proposées (comme “Quelles sont vos vacances idéales?”) ne correspondent pas aux descripteurs de niveau B2, en particulier dans leur adaptation à la communication universitaire comme dans le PEL CercleS. La focalisation sur le calibrage de la matière verbale mène à un manque d'attention sur le processus d'interaction langagière mis en jeu pour le recueil du matériel à évaluer

Les discours émis dans ce cadre ne sont acceptables que dans le contexte d'un exercice scolaire, or Bourdieu mettait déjà en évidence que l'acceptabilité sociale d'un discours ne se limite pas à sa seule grammaticalité. Tenir la parole pendant dix minutes pour "déballer" une opinion mal assurée et peu documentée sur un sujet n'intéressant pas l'interlocuteur serait même socialement inacceptable selon les maximes conversationnelles de Grice (1979). En situation authentique, les stratégies pour avoir "voix au chapitre" sont bien différentes.

\subsection{Analyse de l'oral du DELF B2}

Comme épreuve de production orale niveau DELF B2, il est demandé au candidat de : "Présenter et défendre un point de vue à partir d'un document déclencheur et débattre avec l'examinateur”. Cette consigne est explicitée dans le manuel édité par le CIEP à la rubrique "Conseil pour s'entraîner: développer une argumentation claire et logique":

Il s'agit d'exprimer votre opinion à partir du sujet qui vous est donné. Pour ce faire, vous devez savoir:

- définir une problématique et rechercher des idées

- construire un plan d'exposé,

- articuler votre discours grâce à l'introduction, la conclusion et les transitions.

(Centre International d'Etudes Pédagogiques 2006)

On voit que cette épreuve diffère totalement des conditions proposées lors du séminaire de calibrage. Il s'agit ici d'une épreuve qui relie l'expression orale au traitement d'un texte, ce qui correspond mieux aux pratiques académiques, mais

2 Des exemples de production orale illustrant les niveaux de compétence sont accessibles sur le site http://www.ciep.fr/en/publi_evalcert/dvd-productions-orales-cecrl/index.php 
ne respecte pas le principe affiché d'évaluation distincte des quatre habiletés. Un enjeu communicatif se met en place : montrer à l'autre que l'on a compris un texte provenant de sa société et qu'on est capable d'émettre une opinion sur un élément du débat social. Les stratégies de production et d'interaction font bien partie de l'évaluation. Toutefois cette épreuve ne nous satisfait pas sous plusieurs aspects.

D’un point de vue interculturel, plusieurs choix apparaissent discutables. En effet, le candidat est obligé:

- d'assumer le topic proposé dans la presse francophone comme propre alors qu'il n'en partage pas forcément l'implicite culturel;

- de se plier à un exercice de mimétisme de pratiques langagières caractéristiques du discours scolaire français avec sa surreprésentation du discours argumentatif ;

- de subir un entretien contradictoire sans que l'enjeu du débat soit la co-construction de sens autour du topic, puisque l'examinateur se dérobe quant à l'expression de sa propre opinion et ne fait que jouer l'avocat du diable afin de provoquer une performance lui permettant de vérifier les compétences fonctionnelles du candidat pour réaliser l'acte de parole "défendre son opinion" ;

- de se soumettre à une culture de l'évaluation parfois fort différente de celle de son milieu de socialisation scolaire, d'où des difficultés de gestion du stress dû aux difficultés de maintien de la face (Goffman 1967) en l'absence de tout étayage documentaire (programme à préparer, dossier à présenter, etc.).

La linguistique socio-interactionnelle nous fournit deux indications à prendre en compte (Mondada et Pekarek 2000):

- les compétences langagières ne sont pas indépendantes des conditions socio-interactionnelles où elles sont mobilisées pour la production d'une performance

- tout discours se définit comme co-construction où s'organise le lien social, d'où le caractère local et à la fois socio-historique de l'interaction.

Dans notre cas, si l'on tient à vérifier la disponibilité de moyens linguistiques permettant au sujet de se lancer dans un développement argumentatif, un premier pas est de vérifier que le candidat dispose de matériaux sémantiques à mettre en discours et que l'interaction répond à des critères de vraisemblance fonctionnelle. Les candidats dans leur détresse communicative sur l'objet du débat ne sont pas dans la meilleure posture pour penser à manier les indicateurs 
extérieurs d'une pensée dialectique (phrases élaborées, connecteurs logiques). Dans ces conditions la performance est-elle représentative de la compétence?

Des choix qui relèvent de la politique linguistique sont à faire si l'on considère l'interaction en situation de certification comme partie prenante des rapports socioculturels qui s'instaurent entre des communautés linguistiques ou nationales différentes. Le dialogue du candidat avec l'examinateur est souvent, en contexte où l'enseignement est confié à des non-natifs, le premier contact avec un ressortissant de la communauté étrangère ; ce rite de passage prend valeur d'inauguration d'un rapport avec cette société. L'examinateur et son institution doivent donc se demander quelle culture communicative ils veulent instaurer en tant que locuteur natif avec les sujets sociaux qui souhaitent se ménager un accès à leur communauté de parole ? Dans notre cas : quel accueil ménage-t-on aux nouveaux locuteurs de l'espace francophone?

Or sur ce choix il y a convergence de fond entre les choix du Conseil de l'Europe et ceux de la francophonie : l'option du dialogue interculturel comme modalité et finalité éducative en accord avec un certain projet politico-culturel. Il y a aussi convergence avec le projet humaniste propre à la formation universitaire.

\section{Pour un recentrage de l'évaluation sur le sujet apprenant plurilingue grâce au PEL, instrument de médiation entre standard et contexte}

Le PEL présente selon nous un lieu précieux de médiation entre une nécessaire standardisation des processus de certifications qui engendrent leur valeur transférable, et une non moins nécessaire contextualisation du protocole dans la dimension sociale et interculturelle de l'apprentissage.

Le diplôme photographie un état des compétences, le PEL inscrit cet instant dans une dimension temporelle dynamique par enregistrement des traces d'apprentissage comme par inscription des futurs objectifs. On utilise le PEL crayon et gomme à la main, on retire et rajoute des documents qui inscrivent l'apprentissage dans son contexte vécu, c'est un espace de parole, c'est l'inverse du diplôme qui fixe la compétence abstraite en langue. Nous misons sur l'alliance de ces deux approches complémentaires.

Par ailleurs les études (Hamp-Lyons and Condon 2000) sur les approches d'évaluation en langue avec portfolio, indiquent bien qu'une des premières questions des apprenants est “qui lira mon portfolio ?”. Une instance de réception, une forme de retour (feedback) doit être envisagée pour justifier l'investissement 
en temps engendré par le suivi du portfolio. L'autoévaluation, l'autonomie ne se suffisent pas à elles-mêmes.

Le dispositif mis au point lors de notre session expérimentale DELF et Portfolio niveau B2 en juin 2009 à l'Université de Macerata, prévoyait donc la passation d'un oral sur présentation du Portfolio PEL avec la biographie langagière et le dossier complétés en vue de l'entretien oral pendant la formation spécifique au CLA de l'Université. Examinons les atouts de cette formule.

\subsection{De l'autoévaluation à la certification : un parcours de réussite}

On sait que le PEL est un instrument personnel de l'apprenant, il ne saurait donc être évalué en tant que tel, mais il constitue dans notre projet un support qui accompagne le candidat tout au long d'un processus d'apprentissage qui ne s'arrête pas au jour de la passation d'examen. Utiliser le PEL en situation d'examen permet donc de resituer un moment et un échantillon ponctuels dans un contexte plus vaste.

Les listes de repérage des compétences permettent ainsi à la personne de composer son programme d'apprentissage en se fixant des objectifs puis d'évaluer par elle-même (avec l'aide de son enseignant) si elle est parvenue au niveau ciblé. Les éléments du dossier sont aussi là pour étayer l'autoévaluation. Le candidat à la certification ne se présente donc pas sur un pari, mais choisit le niveau et la session en étant conscient de ses capacités et limites. Cet aspect est extrêmement important pour limiter drastiquement un échec qui est inacceptable pour un diplôme payant dans le contexte éducatif public. Le processus continu de l'autoévaluation, essentiel pour la réussite, avec toutes ses potentialités et problématiques (Little 2005), précède celui de l'évaluation sommative qui vient conclure un parcours en contexte éducatif qui a commencé bien avant la salle d'examen.

\subsection{L’autobiographie langagière pour une éducation au plurilinguisme}

La première partie de l'épreuve se fonde sur la biographie langagière du candidat qui est donc invité à se présenter du point de vue de son engagement dans la diversité linguistique. Cette tâche va au-delà d'une simple prise de contact car elle sollicite en amont un travail de réflexion sur le croisement des langues avec le parcours biographique, la diversité des pratiques sociales dans les différentes 
langues, les différentes approches et méthodologies d'apprentissage. Le riche vécu des candidats est convoqué mais avec le filtre de son versant linguistique qui permet de ne pas forcer l'intimité en ne sollicitant que ce que le candidat a choisi d'exposer en public lors de sa préparation. Cette tâche permet de recueillir une narration située, toujours mêlée d'évaluations subjectives, d'expression de causes, conséquences et finalités ; elle fournit donc des matériaux utiles aux fins de l'évaluation linguistique. Mais elle participe aussi aux finalités d'une éducation linguistique au sens large en permettant l'émergence des profils plurilingues, en favorisant la conscience métalangagière, en valorisant la pratique plurilingue.

On note cependant que le PEL CercleS propose des fiches pédagogiques qui compartimentent la biographie en fiches par langue, ce qui ne favorise pas la construction d'une compétence plurielle.

On désignera par compétence plurilingue et pluriculturelle, la compétence à communiquer langagièrement et à interagir culturellement d'un acteur social qui possède, à des degrés divers, la maîtrise de plusieurs langues et l'expérience de plusieurs cultures. On considérera qu'il n'y a pas là superposition ou juxtaposition de compétences distinctes, mais bien existence d'une compétence complexe, voire composite, dans laquelle l'utilisateur peut puiser. (Conseil de l'Europe 2001: 129).

Par ailleurs ces fiches séparent les langues en particulier entre langues apprises (légitimées par l'école) et langues “patrimoniales”, cette division ne repose sur aucun critère scientifiquement acceptable.

La didactique de la biographie linguistique est une piste ouverte (Zarate et al. 2008) qui reste à décliner dans des activités pédagogiquement pertinentes.

\subsection{Le dossier : construire un lien personnel avec la langue-culture}

La deuxième partie de cette nouvelle épreuve consiste en un bref exposé du candidat sur un thème de son choix préparé dans le dossier du PEL avec une série de documents d'appui. Le calibrage de cette épreuve est délicat, car il ne s'agit pas de produire une performance de type “exposé académique” qui demanderait plus de temps de parole ; un discours trop spécialisé n’aurait d'ailleurs pas d'instance de réception adéquate. On considère aussi que la production de discours argumentatif est déjà évaluée à l'écrit, en particulier la maîtrise de l'organisation textuelle et des articulateurs du discours.

Il s'agit donc plutôt d'un compte-rendu d'une recherche personnelle sur documents de presse, romans, films ou essais. Le candidat choisit un produit 
culturel auquel l'apprentissage du français lui a donné accès. Il témoigne ainsi d'une fréquentation personnalisée du débat social ou de la scène culturelle chez l'autre ; le candidat est invité à exposer son intérêt pour le thème, y compris d'un point de vue comparatif. Puis la conversation s'engagera avec l'examinateur suivant ce qu'il jugera intéressant d'approfondir. Il ne s'agira pas d'un entretien contradictoire, mais d'une exposition d'un point de vue informé, apte à susciter l'intérêt de l'examinateur en tant que natif et expert interculturel toujours intéressé par la vision des étrangers sur sa propre communauté, par la construction en commun de sens dans des pratiques culturelles situées.

On n'aboutira certes pas au "bonheur conversationnel” (Auchlin 1996) car l'entretien subit la tension de son niveau transactionnel primaire avec pour enjeu la délivrance du diplôme, mais du moins cette épreuve inaugurera un accueil de la parole, par une rencontre brève mais authentique avec un médiateur autorisé. Cette interaction ouvre aussi des voies sur la prise en compte des compétences interculturelles dans l'évaluation (Helm and Davies, 2010). Une analyse en linguistique interactionnelle du corpus enregistré lors de la session expérimentales a été réalisée, elle confirme notre hypothèse (Anquetil 2010) mais la validation du dispositif par le CIEP est encore en cours de négociation ${ }^{3}$.

\section{Conclusion}

Nous avons donc par cette expérience tenté de mettre en place une collaboration complémentaire entre la démarche Portfolio et celle de l'évaluation certificative, une collaboration entre plusieurs institutions avec leurs visées respectives: le PEL dans les finalités européennes du Lifelong Learning Programme, l'agence gouvernementale française de certifications dans le souci d'un rayonnement culturel de la langue française et l'université, en particulier son centre linguistique, dans sa perspective conjuguant recherche didactique et scientifique, et intervention contextualisée, ancrée dans un terrain, à l'écoute des acteurs impliqués dans les dispositifs.

Passer une certification ressemble parfois à un parcours initiatique risqué de mise à des normes standardisées de la parole pour entrer dans un groupe exclusif, or nous voudrions changer de paradigme pour un parcours de "reconnaissance”, un mot dont la polysémie nous mène en français jusqu'à la philosophie de l'action de Paul Ricœur (2004).

3 En 2010-2011. 
L'examinateur reconnaît la compétence sous la performance mais il la reconnaît dans une parole contextuelle ; il reconnaît aussi:

- la demande de reconnaissance du candidat au vu de son effort vers la langue-culture de l'autre,

- la recherche d'une identité culturelle plus ample et complexe, faisant propres certains aspects de la culture de l'autre, mais par réinterprétation personnelle.

Il reconnaît, derrière le PEL, le travail d'un système éducatif et d'un enseignant qui s'est mis de côté et qui ne recueille qu'indirectement le succès de son élève. La certification est une relation sociale engageante, pas seulement un geste technique.

\section{Bibliographie}

Anquetil, Mathilde. 2010. L'interaction en situation de certification de FLE, un regard critique. Colloque international "Spécificités et diversité des interactions didactiques : disciplines, finalités, contextes”, Université de Lyon - ICAR - CNRS - INRP, 24-26 juin 2010, LYON, INRP, France. http://halshs.archives-ouvertes.fr (24.06.2010, mis en ligne le 09.02.2011).

Anquetil, Mathilde \& Marie-Christine Jamet. 2010. Intégrer les certifications dans un cursus universitaire: enjeux, défis, interrogations. Revue française de linguistique appliquée 15(1). 61-74.

Auchlin, Antoine. 1996. Bonheur conversationnel et qualité du discours: pour une approche expérientielle et systémique de la compétence discursive. Langage et Pratiques (Lausanne: Bulletin de l'ARLD). 13-17.

Bourdieu, Pierre. 1982. Ce que parler veut dire. L'économie des échanges linguistiques. Paris: Fayard.

Byram, Michael \& Geneviève Zarate. 1997. La compétence socio-culturelle dans l'apprentissage et l'enseignement des langues. Strasbourg : Editions du Conseil de l'Europe.

Centre International d’Etudes Pédagogiques. 2006. Réussir le DELF niveau B2 du Cadre Européen Européen Commun de Référence. Paris : Didier.

Conseil de l'Europe. 2001. Cadre européen commun de référence pour les langues: apprendre, enseigner, évaluer. Paris: Didier.

Goffman, Erving. 1967. Les rites d'interaction. Paris: Editions de Minuit.

Grice, H. Paul. 1979. Logique et conversation. Communications 30. 57-72.

Hamp-Lyons, Liz \& William Condon. 2000. Assessing the portfolio: principles for practice, theory and research. Cresskill NJ: Hampton Press.

Helm, Francesca \& Gillian Davies. 2010. Computer-mediated communication, electronic diaries and the European Language Portfolio for self, peer and tutor assessment of intercultural competences. In Fred Dervin \& Eija Suomela-Salmi (eds.), New approaches to assessing language and (inter-)cultural competences in higher education, 193-206. Frankfurt am Main: Peter Lang. 
Kern, Richard \& Anthony Liddicoat. 2008. In Geneviève Zarate, Danielle Lévy \& Claire Kramsch (eds.). 2008. Précis du plurilinguisme et du pluriculturalisme. Paris: Editions des archives contemporaines. 27-33.

Kohonen, Viljo. 2001. The European Language Portfolio: from portfolio assessment to portfoliooriented language learning. In Viljo Kohonen, Riitta Jaatinen, Pauli Kaikkonen \& Jorma Lehtovaara (eds.), Experiential learning in foreign language education, 8-60. London: Pearson Education.

Lévy, Danielle. 2001. Les implications du changement social dans l'éducation linguistique à l'université. In Françoise Bidaud, Lingue e culture romanze, didattica e ricerca : quali prospettive, Atti del convegno DORIF-Università, 1-22. Viareggio-Lucca: Mauro Baroni Editore.

Lévy, Danielle. 2008. Soi et les langues. In Geneviève Zarate, Danielle Lévy \& Claire Kramsch (eds.). 2008. Précis du plurilinguisme et du pluriculturalisme. Paris: Editions des archives contemporaines. 69-81.

Little, David. 2005. The Common European Framework and the European Language Portfolio: involving learners and their judgements in the assessment process. Language Testing 22(3). 321-336.

McNamara, Tim \& Carsten Roever. 2006. Language testing: The social dimension. Oxford: Blackwell.

McNamara, Tim. 2010. The use of language tests in the service of policy : issues of validity. Revue Française de Linguistique Appliquée, Evaluer les compétences langagières, XV-1. 7-23.

Messick, Samuel. 1989. Validity. In Linn, R.L. (ed), Educational measurement (3 $3^{\text {rd }}$ ed.). New york : American Council on Eucation \& Macmillan. 13-103.

Mondada, Lorenza \& Simona Pekarek Doehler. 2000. Interaction sociale et cognition située : quels modèles pour la recherche sur l'acquisition des langues? Acquisition et Interaction en Langue Etrangère 12. http://aile.revues.org (mis en ligne le 09 novembre 2010).

Quinghua, Li. 2010. The impact of portfolio-based writing assessment on EFL writing development of Chinese learners. Chinese Journal of Applied Linguistics 33(2), 103-116. http://www.celea.org.cn/teic/90/10060808.pdf (consulté le 20.02.2012).

Ricœur, Paul. 2004. Parcours de la reconnaissance. Paris: Stock.

Shohamy, Elana. 2001. The power of tests : A critical perspective on the uses of language tests. Harlow: Pearson Education.

\title{
Annexe ${ }^{0} 1$ : Projet expérimental DELF B2 et Portfolio. Consignes aux candidats
}

\author{
DELF B2 et Portfolio \\ Déroulement de l'épreuve de Production orale \\ Macerata - 3 juin 2009
}




\section{Déroulement horaire}

1. Préparation épreuve CIEP (sujet tiré au sort) (30 mn)

2. Passation épreuve CIEP (15-20 mn)

3. Epreuve sur Portfolio (15-20 mn)

\section{Déroulement de l’épreuve Portfolio}

\section{Présentation du Portfolio}

1.1. Bonjour ! Pouvez-vous vous présenter? Avez-vous préparé votre CV ?

1.2. Vous avez préparé un profil langagier dans le passeport Européen des langues, commentez-le. L'usage du portfolio et de ses descripteurs de compétences vous a-t-il permis de faire le point sur vos compétences en langues, de fixer des objectifs?

1.3. Vous avez réfléchi sur votre biographie langagière : pouvez-vous parler de votre apprentissage des langues, comment les avez-vous apprises, quelles sont vos expériences marquantes (en positif ou en négatif) avec les langues et les cultures étrangères ? Pourquoi et comment apprenez-vous le français ?

1.4. Quels documents avez-vous insérés dans votre dossier comme documentation sur votre apprentissage du français et vos compétences? Pourquoi les avez-vous choisis?

\section{Présentation du dossier thématique pour l'épreuve}

2.1. Vous avez choisi de présenter un sujet, lequel ? Pourquoi ?

2.2. Exposez votre recherche.

2.3. Débat : l'examinateur va maintenant vous poser des questions,

- pour préciser et expliciter certains points ...

- pour vous demander d'argumenter afin de défendre votre opinion ou de la nuancer en prenant en compte une critique ou un argument divergent ...

\section{$\underline{\text { Conseils }}$}

Préparez-vous mais ne lisez pas, n’apprenez pas par cœur votre texte. Parlez pour informer clairement et convaincre votre auditoire. Montrez que vous avez des 
connaissances sur le thème traité, que vous vous êtes informé, que vous avez réfléchi à la question; comparez à la situation en Italie si le sujet s'y prête. N'oubliez pas que l'examinateur ne connaît pas votre sujet, c'est vous qui construisez le thème de la conversation!

\section{Annexe $\mathrm{n}^{\circ} 2$ : Projet expérimental DELF B2 et Portfolio : critères d'évaluation}

\section{DELF B2 et Portfolio}

Grille d'évaluation de la Production orale sur Portfolio

\section{Compétences communicatives}

\begin{tabular}{|l|l|l|l|l|l|l|l|l|l|}
\hline $\begin{array}{l}\text { 1ère partie - présentation du Portfolio Européen } \\
\text { des Langues }\end{array}$ & & & & & & & & & \\
\hline $\begin{array}{l}\text { peut présenter sa biographie langagière } \\
\text { (parcours d'apprentissage, profil et objectifs) } \\
\text { de manière structurée }\end{array}$ & 0 & 0.5 & 1 & 1,5 & 2 & & & & \\
\hline $\begin{array}{l}\text { peut présenter et mettre en valeur les éléments } \\
\text { constitutifs de son dossier }\end{array}$ & 0 & 0,5 & 1 & & & & & & \\
\hline $\begin{array}{l}\text { peut exposer clairement un point de vue } \\
\text { organisé en pertinence avec les documents } \\
\text { choisis en vue de l'épreuve }\end{array}$ & 0 & 0,5 & 1 & 1,5 & 2 & 2,5 & 3 & & \\
\hline
\end{tabular}

\begin{tabular}{|l|l|l|l|l|l|l|l|l|l|}
\hline $\mathbf{2}^{\mathbf{2}^{\mathrm{m} e}}$ partie - interaction & & & & & & & & \\
\hline $\begin{array}{l}\text { peut, à la demande, apporter des précisions, } \\
\text { confirmer son opinion en introduisant des } \\
\text { éléments significatifs, des arguments ou } \\
\text { exemples pertinents }\end{array}$ & 0 & 0.5 & 1 & 1,5 & 2 & 2,5 & & & \\
\hline $\begin{array}{l}\text { peut réagir à un argument critique pour défendre } \\
\text { sa position ou évoluer dans son opinion }\end{array}$ & 0 & 0,5 & 1 & 1,5 & 2 & 2,5 & & \\
\hline
\end{tabular}




\section{Qualité linguistique pour l'ensemble de l'épreuve}

\begin{tabular}{|c|c|c|c|c|c|c|c|c|c|}
\hline $\begin{array}{l}\text { Phonologie } \\
\text { a acquis une prononciation et une } \\
\text { intonation claire }\end{array}$ & 0 & 0,5 & 1 & 1,5 & 2 & & & & \\
\hline $\begin{array}{l}\text { Morphosyntaxe } \\
\text { a un bon contrôle grammatical malgré } \\
\text { quelques erreurs n'affectant pas le sens }\end{array}$ & 0 & 0.5 & 1 & 1,5 & 2 & 2,5 & 3 & 3,5 & 4 \\
\hline $\begin{array}{l}\text { Compétence discursive } \\
\text { montre une bonne maîtrise des outils } \\
\text { d'organisation du discours }\end{array}$ & 0 & 0,5 & 1 & 1,5 & 2 & 2,5 & 3 & & \\
\hline $\begin{array}{l}\text { Lexique } \\
\text { possède une bonne variété de vocabulaire } \\
\text { même si des lacunes subsistent }\end{array}$ & 0 & 0,5 & 1 & 1,5 & 2 & & & & \\
\hline
\end{tabular}

\section{Compétences socio-culturelles}

\begin{tabular}{|l|l|l|l|l|l|l|l|l|}
\hline $\begin{array}{l}\text { Savoirs socio-culturels } \\
\text { témoigne d'une bonne capacité d'interprétation des } \\
\text { aspects socio-culturels liés à la thématique } \\
\text { présentée (éventuellement dans une perspective } \\
\text { comparative) }\end{array}$ & 0 & 0,5 & 1 & 1,5 & 2 & & & \\
\hline $\begin{array}{l}\text { Compétenes sociolinguistiques } \\
\text { sait commencer, soutenir et terminer l'interaction } \\
\text { de façon adéquate (formules de politesse, registre } \\
\text { de langue, prise de parole, congé ...) }\end{array}$ & 0 & 0.5 & 1 & & & & & \\
\hline
\end{tabular}

\section{Bionote}

Mathilde Anquetil est enseignant-chercheur de Langue Française auprès de la Faculté de Sciences Politiques de l'Université de Macerata (Italie). 